\title{
La relación entre las empresas españolas y el CSIC: motivaciones, mecanismos y beneficios desde la perspectiva empresarial
}

Oihana Valmaseda-Andia*, Eneka Albizu-Gallastegi**, Manuel Fernández-Esquinas***, Ignacio Fernández-de-Lucio****

\author{
* Universidad del País Vasco/Euskal Herriko Unibertsitatea UPV/EHU. Correo-e: oihana.valmaseda@ehu.es \\ ** Universidad del País Vasco/Euskal Herriko Unibertsitatea UPV/EHU. Correo-e: eneka.albizu@ehu.es \\ *** Instituto de Estudios Sociales Avanzados (CSIC). Correo-e: mfernandez@iesa.csic.es \\ **** INGENIO (CSIC-Universitat Politècnica de València). Correo-e: ifernand@ingenio.upv.es
}

Recibido: 09-02-2015; 2a versión: 14-04-2015; Aceptado: 23-04-2015.

Cómo citar este artículo/Citation: Valmaseda-Andia, O.; Albizu-Gallastegi, E.; Fernández-Esquinas, M.; Fernández-de-Lucio, I. (2015). La relación entre las empresas españolas y el CSIC: motivaciones, mecanismos y beneficios desde la perspectiva empresarial. Revista Española de Documentación Científica, 38(4): e109. doi: http://dx.doi.org/10.3989/redc.2015.4.1263

\begin{abstract}
Resumen: El presente trabajo explora las motivaciones por las que las empresas españolas interaccionan con el Consejo Superior de Investigaciones Científicas (CSIC), describe los mecanismos que relacionan a ambos agentes, y, por último, explica el tipo de beneficio que han obtenido como producto de esta relación. Para ello, se analiza la información facilitada por 787 empresas españolas que han trabajado con el CSIC en actividades de innovación durante el periodo 1999-2010. El estudio permite concluir que las empresas, para relacionarse con el CSIC, prefieren y valoran mejor las motivaciones estratégicas que las tácticas, y en la relación utilizan una amplia variedad de mecanismos entre los que destacan los de apoyo tecnológico o consultoría y los de investigación contratada, mecanismos con bajo nivel de sofisticación. Además, los beneficios que las empresas obtienen rebasan los efectos sobre la innovación y, en consecuencia, sobre los beneficios económicos asociados a la misma, y cubren una amplia gama de resultados tangibles e intangibles.
\end{abstract}

Palabras clave: Relaciones empresa-ciencia; innovación; sistema público de I+D; transferencia de conocimiento; organismo público de investigación (OPI).

Interaction between the Spanish firms and the CSIC: motivations, mechanisms and benefits from the firm's perspective

Abstract: This paper explores Spanish firms' motivations to interact with the Spanish National Research Council (CSIC) and describes the mechanisms by which both agents are related. Finally, it also explores the type of benefit obtained as a result of this interaction. To this aim, we examine data provided by 787 Spanish companies which took part with the CSIC in innovation activities during the period 1999-2010. The study points out that Spanish firms prefer strategic motivations than tactical ones, and that the major mechanisms underlying such interactions are technological support or consulting and contract research. That is, all of them mechanisms with low level of sophistication. Finally, we observe that the benefit obtained by firms goes beyond innovation, or even economical profit associated with it. Different types of tangible and intangible benefits are described in detail.

Keywords: Science-industry interaction; innovation; public R\&D system; knowledge transfer; public research organization (PRO).

Copyright: (C) 2015 CSIC. Este es un artículo de acceso abierto distribuido bajo los términos de la licencia Creative Commons Attribution-Non Commercial (by-nc) Spain 3.0. 


\section{INTRODUCCIÓN}

Los desafíos a los que se enfrenta la sociedad requieren, más que nunca, del conocimiento científico y tecnológico, siendo necesaria para su creación la intervención y cooperación, tanto de los actores de la comunidad científica, como del resto de los ámbitos económicos y sociales. Este escenario de interacción entre agentes es el que propicia las condiciones bajo las cuales las empresas encuentran una vía para desarrollar su actividad innovadora.

Si bien la mayor tendencia de las empresas a cooperar con instituciones científicas, y la efectividad de dichas relaciones, están crecientemente documentadas por la investigación realizada hasta la actualidad, persisten aún ciertas limitaciones en este campo. Por un lado, la práctica inexistencia de estudios que se ocupen específicamente del papel que desempeñan los organismos públicos de investigación. Estas instituciones representan una parte importante de las estructuras de investigación existentes en España y se caracterizan por tener finalidades, así como dinámicas de actuación y organización, que difieren de otras organizaciones como las universidades, centros tecnológicos, etc.

Por otro lado, en un segmento importante de los trabajos de investigación existentes (i.e. Jaffe, 1989; Mowery y Ziedonis, 2002; Thursby y Thursby, 2002; Di Gregorio y Shane, 2003), ha predominado el análisis de determinados mecanismos de transferencia de conocimiento, como por ejemplo, la licencia de patentes y la creación de spin-offs. Sin embargo, el acceso al conocimiento de los centros de investigación por parte del tejido empresarial parece producirse a través de un mapa variado de mecanismos, en el que estas fórmulas no tienen necesariamente un papel protagonista (Agrawal, 2001; Cohen y otros., 2002; D'Este y Patel, 2007; Perkmann y Walsh, 2007; Bekkers y Boda-Freitas, 2008; Arza, 2010; MerchánHernández, 2012; García-Carpintero y otros, 2014).

Finalmente, la evaluación del efecto de la interacción entre el sector empresarial y científico es, sin duda, una de las cuestiones esenciales a resolver en este campo, ya que no se dispone aún de una visión integral de la efectividad de la investigación pública en la actividad empresarial. Así, en una parte de los estudios existentes hasta el momento es manifiestamente visible la preocupación por el uso de medidas de impacto económico o de valor añadido, cuando en realidad, los efectos de la interacción público-privada son observables en distintos ámbitos de la empresa (Barge-Gil y Modrego, 2011; De Fuentes y Dutrénit, 2012), trascendiendo, por tanto, a esta mirada reduccionista.
Tomando en consideración las mencionadas limitaciones, el objeto del presente trabajo se centra, específicamente, en el estudio de un caso particular de interacción para la innovación, es decir, la que se produce entre las empresas españolas y los institutos de investigación del Consejo Superior de Investigaciones Científicas (CSIC). Sobre dicho caso particular, y a partir de los datos recabados de 787 empresas españolas que han colaborado con este organismo, se examinan tres cuestiones de gran relevancia: ¿por qué las empresas españolas se relacionan con el CSIC?, ¿cómo cristaliza esa relación? y ¿qué resultados empresariales se derivan de la misma?.

Conviene poner en valor la fuente de datos utilizada, tanto por su especificidad y tamaño, como por su carácter inédito. En la presente investigación se accede y analizan por primera vez las empresas españolas que han trabajado con el CSIC en el periodo 1999-2010. Este planteamiento constituye una aportación original con respecto a las investigaciones que asientan sus conclusiones en encuestas de carácter general, como por ejemplo, las encuestas sobre innovación y actividades de I+D que, sistemáticamente, realizan organismos como las agencias nacionales de estadística. Estas encuestas ofrecen una información limitada sobre la interacción entre agentes.

El contenido del artículo se estructura de la siguiente forma. En la parte inicial, se recogen los principales antecedentes de la literatura que han analizado las motivaciones de las empresas para la interacción con agentes externos, los mecanismos bajo los cuales se materializa la interacción y los resultados que obtienen las empresas de la misma. A continuación, se explica el procedimiento metodológico empleado, como también la fuente de datos utilizada en la investigación. Seguidamente, se pone el foco en el estudio del contenido de la relación entre las empresas españolas y el CSIC. El trabajo concluye con un apartado en el que se recogen las principales conclusiones del estudio.

\section{MOTIVACIONES, MECANISMOS Y BENEFI- CIOS DE LAS RELACIONES ENTRE LAS EMPRE- SAS Y EL SECTOR CIENTÍFICO: PERSPECTIVA EMPRESARIAL}

\subsection{La dimensión motivacional de la interac- ción entre el sector empresarial y el científico}

El enfoque de los sistemas de innovación (Lundvall, 1992; Edquist, 1997) considera la innovación como un proceso dinámico y social basado en el aprendizaje y en la interacción entre los diversos 
agentes, así como el reconocimiento del carácter endógeno del cambio tecnológico y la capacidad del propio sistema económico para controlarlo y dirigirlo. A este respecto, las empresas innovadoras necesitan acceder a los recursos de conocimiento que generan los otros agentes del sistema de innovación (SI), entre los que se encuentran las organizaciones públicas de investigación. Los motivos para acceder a estas fuentes externas del conocimiento son diversos (Vega y otros, 2009). Uno de ellos es identificar y explotar las tecnologías externas que puedan significar nuevas oportunidades para la empresa. Otras motivaciones tienen que ver con cuestiones puramente económicas como el ahorro en los costes de desarrollo de determinadas actividades (Bonaccorsi y Piccaluga, 1994) o la obtención de financiación adicional para proyectos (Bayona y otros, 2002).

Algunos de estos motivos son propios de estrategias diseñadas para horizontes temporales cortos, y encaminadas a resolver, fundamentalmente, problemas de producción. En definitiva, se trata de un patrón de comportamiento que podría ser calificado de "pasivo", frente a otro tipo de estrategia construida sobre razones de índole intelectual, y orientada a la obtención de conocimiento científico cercano a las fronteras del conocimiento (Arza, 2010).

En el contexto español, entre los estudios más recientes destaca el trabajo de Barge-Gil y Modrego (2011) quienes distinguen dos tipos de motivaciones: las tecnológicas y las económicas. Las razones económicas están relacionadas con la reducción de costes y riesgos económicos (Veugelers, 1998; Bayona y otros, 2001; Geisler, 2001; Miotti y Sachwald, 2003; Becker y Dietz, 2004; Narula, 2004). En particular, BargeGil y Modrego (2011) incluyen en su estudio la reducción de los costes de los proyectos, la reducción del riesgo asociado a los proyectos, el cumplimiento de las condiciones de los programas públicos y la mejora de la reputación, en el plano económico. Las motivaciones tecnológicas son aquellas encaminadas al acceso de recursos complementarios, generalmente conocimiento y experiencia que la empresa no posee, y que requiere para desarrollar actividades innovadoras. De acuerdo con esta definición, los autores introducen en su estudio la adquisición de conocimiento o experiencia, la monitorización o seguimiento de los avances tecnológicos y el acceso a infraestructuras de investigación como ejemplos de motivaciones tecnológicas.
Gutiérrez-Gracia y otros (2010), reconocen dos tipos de motivaciones para la interacción: las motivaciones tácticas y las estratégicas. Las motivaciones tácticas se relacionan con la reducción de riesgos y costes asociados a las actividades de innovación y que se circunscriben a horizontes temporales cortos. Las motivaciones estratégicas son las que se asocian con el acceso a recursos y capacidades complementarios del agente con el que se coopera, y cuyo alcance es del largo plazo (Hagedoorn, 1993; Hagedoorn y otros, 2000).

Merchán-Hernández (2012), para el caso de la región de Andalucía, señala que entre las motivaciones del sector empresarial para la interacción con las universidades destacan, en primer lugar, el acceso a redes de conocimiento científico y técnico no disponibles en el sector empresarial y, en segundo lugar, la posibilidad de adelantarse a los cambios técnicos que puedan darse en un sector concreto, seguido del acceso a personas de la universidad, que a futuro, puedan formar parte de la plantilla de personal de la empresa.

En síntesis, estas perspectivas analíticas configuran un mapa amplio y diverso de las motivaciones que guían a las empresas en su relación con la comunidad científica.

\subsection{Los mecanismos que articulan la interac- ción entre el sector empresarial y el científico}

La evidencia empírica existente sugiere que entre las empresas y el sector público de investigación se realizan intercambios de diversos objetos tales como el conocimiento científico, técnicas, aparatos, know-how, etc., (Bozeman y otros, 1995), a través de mecanismos también variados, siendo los más ampliamente reconocidos la formación, la consultoría, los contratos de I+D, los proyectos de investigación conjunta, la movilidad de recursos humanos, la participación conjunta en publicaciones y conferencias, y la comercialización de los resultados de la investigación.

La literatura especializada ha tratado esta diversidad de mecanismos acudiendo a diferenciaciones entre ellos que tienen implicaciones para las dinámicas de transferencia de conocimiento entre el sector científico y el empresarial. Una primera diferencia se refiere al grado de formalización de las relaciones. A saber, el grado en que las interacciones están institucionalizadas o guiadas por reglas y procedimientos formales, así como la permanencia en el tiempo de dicha institucionalización (Ponomariov y Boardman, 2012). 
Una segunda diferencia importante relacionada con la anterior es el grado de intensidad de las relaciones (Perkmann y Walsh, 2007). Algunos tipos de mecanismos van asociados inherentemente a una participación personal de las partes implicadas. Mecanismos que suelen requerir un nivel de interacción alto son la investigación contratada y los proyectos cooperativos. El tipo de implicación suele ir asociado al carácter tácito del conocimiento que se pretende transferir.

Una tercera división hace referencia a la sofisticación del conocimiento, en el sentido de que existen formas de interacción asociadas a la producción de nuevo conocimiento científico. En estos casos el carácter de los resultados no suele tener un alto grado de "finalización" en un conjunto discreto de resultados tangibles y utilizables (Schartinger y otros, 2002), y no suele existir certidumbre respecto a los logros del nuevo conocimiento a producir. Por el contrario, otros mecanismos de interacción se dirigen a la utilización de conocimiento acumulado para resolver problemas concretos, como es el caso de la consultoría o la prestación de servicios a partir de la utilización de infraestructuras de I+D.

En la Tabla I se recogen, a modo de síntesis, las investigaciones de referencia en el estudio de la diversidad de mecanismos en la vinculación de las empresas con el sector científico.

\subsection{Beneficios de las relaciones desde la perspectiva empresarial}

La literatura especializada en este campo suele clasificar los posibles resultados de la interacción con los organismos públicos de I+D atendiendo a diferentes criterios. Se han identificado resultados de tipo tangible, como la mejora de un producto o servicio, la creación de un prototipo o la generación de una patente, o bien, de naturaleza intangible, como el desarrollo y fortalecimiento del know-how de la empresa, el incremento del conocimiento científico de la misma o la mejora de la cualificación del personal de la empresa. Otros estudios abordan esta cuestión identificando la influencia que la investigación pública tiene en la industria, tratando de observar en qué ámbitos de la empresa se materializa dicha influencia, como por ejemplo, si surgieron nuevos proyectos o ideas, o bien, si contribuyeron a completar dichos proyectos (Cohen y otros, 2002).

Bishop y otros (2011) examinan un rango de posibles beneficios clasificándolos en lo que denominan beneficios de exploración, relacionados con la búsqueda y acceso a conocimiento, y en los beneficios de explotación, más vinculados a resultados de índole comercial que conllevan la explotación de los conocimientos.

La naturaleza multidimensional y compleja del impacto de las relaciones queda manifiesta en el trabajo de Barge-Gil y Modrego (2011), quienes proponen una clasificación que contempla cinco ámbitos de impacto: el impacto técnico, haciendo referencia a resultados de índole técnica directos o inmediatos; el económico, que se refiere a efectos económicos en el corto y medio plazo; el impacto en la inversión de la empresa, es decir, resultados relacionados con la mejora de indicadores de inversión en recursos de la empresa; el impacto intangible, y por último, otros ámbitos de impacto, refiriéndose a medidas cualitativas de afectación.

En la tabla II se resumen los principales referentes conceptuales en el estudio de la tipología de resultados que se derivan de las relaciones entre los agentes empresariales y la comunidad científica.

\section{METODOLOGÍA}

La información de la investigación procede de una encuesta realizada a 1891 empresas españolas que han tenido, al menos, una relación con el CSIC formalizada mediante contrato durante el período 19992010. Respondieron un total de 787 empresas (41\%) que constituyen el objeto de estudio. Se ha observado que son representativas de distintos sectores, tamaños y localización geográfica (véase Tabla III).

Los registros administrativos que tiene el CSIC no recogen medidas sobre el contenido específico y los resultados de los contratos o acuerdos que suscriben con las empresas. Frente a este tipo de problemas, la encuesta realizada ha sido una herramienta fundamental para obtener dicha información. Se eligió el método de entrevista para la recogida de información. Ello obedece a la complejidad y número de dimensiones conceptuales abordadas, que desaconsejaba otros métodos como la encuesta telefónica u on line.

Una de las piezas clave en el proceso de recogida de la información de la encuesta ha sido la identificación, y posterior acceso, a la población operativa del estudio, esto es, aquellas personas que, dentro de la empresa, tienen conocimiento suficiente para valorar el desarrollo y los resultados de la relación de la organización empresarial con el CSIC. El criterio general ha sido seleccionar a los responsables del último proyecto/contrato realizado con el CSIC o, en su defecto, a la persona de la empresa que mayor conocimiento tuviera sobre las actividades de vinculación con el CSIC en el período considerado en el estudio. 
Tabla I. Cuadro resumen de la literatura sobre la variedad de mecanismos que vinculan al sector científico y a las empresas

\begin{tabular}{|c|c|c|c|}
\hline $\begin{array}{l}\text { Referencia } \\
\text { bibliográfica }\end{array}$ & Mecanismos & $\begin{array}{l}\text { Referencia } \\
\text { bibliográfica }\end{array}$ & Mecanismos \\
\hline $\begin{array}{l}\text { Fritsch y } \\
\text { Schwirten } \\
\text { (1999) }\end{array}$ & $\begin{array}{l}\text { Proyectos de investigación conjunta } \\
\text { Contratos de investigación } \\
\text { Consultoría/Informes } \\
\text { Uso conjunto de equipamientos y la- } \\
\text { boratorios } \\
\text { Intercambios de personal y movilidad } \\
\text { Comunicación formal } \\
\text { Contactos informales }\end{array}$ & $\begin{array}{l}\text { Bekkers y } \\
\text { Bodas Freitas } \\
(2008)\end{array}$ & $\begin{array}{l}\text { Investigación colaborativa y contratos de investi- } \\
\text { gación } \\
\text { Patentes y licencias } \\
\text { Movilidad laboral } \\
\text { Contactos a través del alumnado o de organiza- } \\
\text { ciones profesionales } \\
\text { Actividades específicas } \\
\text { Contactos informales y estudiantes graduados }\end{array}$ \\
\hline $\begin{array}{l}\text { Schartinger y } \\
\text { otros (2001) }\end{array}$ & $\begin{array}{l}\text { Proyectos de investigación conjunta } \\
\text { Contratos de investigación } \\
\text { Acuerdos de licencias } \\
\text { Movilidad de investigadores de la uni- } \\
\text { versidad a la empresa } \\
\text { Emplear a graduados } \\
\text { Supervisión conjunta de los trabajos } \\
\text { de tesis } \\
\text { Redes de investigación internacionales }\end{array}$ & Arza (2010) & $\begin{array}{l}\text { Proyectos de investigación conjunta } \\
\text { Contratos de investigación } \\
\text { Consultoría } \\
\text { Uso de equipamiento } \\
\text { Patentes } \\
\text { Licencias de tecnología } \\
\text { Spin-offs } \\
\text { Contratación de graduados } \\
\text { Conferencias } \\
\text { Publicaciones } \\
\text { Formación } \\
\text { Test } \\
\text { Parques científico-tecnológicos } \\
\text { Incubadoras } \\
\text { Participación en redes }\end{array}$ \\
\hline $\begin{array}{l}\text { Cohen y otros } \\
(2002)\end{array}$ & $\begin{array}{l}\text { Contratos de investigación } \\
\text { Consultoría } \\
\text { Patentes } \\
\text { Licencias } \\
\text { Empresas conjuntas y de cooperación } \\
\quad \text { (centros mixtos) } \\
\text { Contratación de recién graduados } \\
\text { Publicaciones e informes } \\
\text { Encuentros y conferencias de carácter } \\
\text { público } \\
\text { Estancias temporales de personal } \\
\text { Intercambios informales de información }\end{array}$ & $\begin{array}{l}\text { Ponomariov } \\
\text { y Boardman } \\
(2012)\end{array}$ & $\begin{array}{l}\text { Proyectos de investigación conjunta o colaborativa } \\
\text { Contratos de investigación } \\
\text { Consultoría académica } \\
\text { Comercialización de derechos de propiedad sobre } \\
\text { resultados de investigación (licencias de paten- } \\
\text { tes y spin-offs) } \\
\text { Creación de centros de investigación cooperativa } \\
\text { o centros mixtos } \\
\text { Contratación de graduados } \\
\text { Intercambios de personal y movilidad } \\
\text { Actividades de difusión (conferencias y publicaciones) } \\
\text { Contactos informales con personal investigador }\end{array}$ \\
\hline $\begin{array}{l}\text { D'Este y Patel } \\
(2007)\end{array}$ & $\begin{array}{l}\text { Investigación conjunta } \\
\text { Consultoría y contratos de investigación } \\
\text { Creación de spin-offs o instalaciones } \\
\text { físicas con financiación empresarial } \\
\text { (laboratorios en un campus, incu- } \\
\text { badoras o centros de investigación } \\
\text { cooperativa) } \\
\text { Formación } \\
\text { Conferencias y encuentros profesionales }\end{array}$ & $\begin{array}{l}\text { De Fuentes } \\
\text { y Dutrénit } \\
(2012)\end{array}$ & $\begin{array}{l}\text { Proyectos de investigación conjunta } \\
\text { Contratos de investigación } \\
\text { Consultoría } \\
\text { Licencias de tecnología } \\
\text { Patentes } \\
\text { Contratación de graduados } \\
\text { Formación } \\
\text { Publicaciones } \\
\text { Conferencias } \\
\text { Contactos informales }\end{array}$ \\
\hline $\begin{array}{l}\text { Perkmann y } \\
\text { Walsh (2007) }\end{array}$ & $\begin{array}{l}\text { Investigación colaborativa } \\
\text { Servicios de investigación } \\
\text { Comercialización de derechos de pro- } \\
\text { piedad intelectual } \\
\text { Emprendimiento } \\
\text { Transferencia de recursos humanos } \\
\text { Publicaciones } \\
\text { Interacción informal }\end{array}$ & $\begin{array}{l}\text { Merchán- } \\
\text { Hernández } \\
\text { (2012) }\end{array}$ & $\begin{array}{l}\text { Investigación conjunta } \\
\text { Investigación contratada } \\
\text { Asesoramiento tecnológico } \\
\text { Alquiler de instalaciones } \\
\text { Explotación de patentes de la universidad o centro } \\
\text { de investigación } \\
\text { Creación de una spin-off } \\
\text { Creación de un centro mixto } \\
\text { Prácticas de personal científico en la empresa } \\
\text { Formación específica por parte de una universidad } \\
\text { o centro de investigación } \\
\text { Relaciones informales }\end{array}$ \\
\hline
\end{tabular}


Tabla II. Resumen de la literatura que aborda los beneficios derivados de la interacción entre las empresas y el sector científico desde la perspectiva empresarial

\begin{tabular}{|c|c|c|c|}
\hline $\begin{array}{l}\text { Referencia } \\
\text { bibliográfica }\end{array}$ & Beneficios de la relación & $\begin{array}{l}\text { Referencia } \\
\text { bibliográfica }\end{array}$ & Beneficios de la relación \\
\hline $\begin{array}{l}\text { Schartinger y } \\
\text { otros (2001) }\end{array}$ & $\begin{array}{l}\text { Acuerdos de licencias } \\
\text { Proyectos de investigación conjunta } \\
\text { Contratos de investigación } \\
\text { Emplear a graduados } \\
\text { Supervisión conjunta de los trabajos de } \\
\text { tesis } \\
\text { Movilidad de investigadores de la univer- } \\
\text { sidad a la empresa }\end{array}$ & $\begin{array}{l}\text { Perkmann y } \\
\text { Walsh (2007) }\end{array}$ & $\begin{array}{l}\text { Comercialización de derechos de propiedad } \\
\text { intelectual } \\
\text { Investigación colaborativa } \\
\text { Servicios de investigación } \\
\text { Publicaciones } \\
\text { Emprendimiento } \\
\text { Transferencia de recursos humanos } \\
\text { Interacción informal }\end{array}$ \\
\hline $\begin{array}{l}\text { Cohen y otros } \\
(2002)\end{array}$ & $\begin{array}{l}\text { Patentes } \\
\text { Contratos de investigación } \\
\text { Consultoría } \\
\text { Publicaciones e informes } \\
\text { Encuentros y conferencias de carácter } \\
\text { público } \\
\text { Empresas conjuntas y de cooperación } \\
\text { (centros mixtos) } \\
\text { Contratación de recién graduados } \\
\text { Intercambios informales de información } \\
\text { Estancias temporales de personal }\end{array}$ & Arza (2010) & $\begin{array}{l}\text { Patentes } \\
\text { Licencias de tecnología } \\
\text { Proyectos de investigación conjunta } \\
\text { Participación en redes } \\
\text { Contratos de investigación } \\
\text { Conferencias } \\
\text { Publicaciones } \\
\text { Consultoría } \\
\text { Uso de equipamiento } \\
\text { Test } \\
\text { Spin-offs } \\
\text { Incubadoras } \\
\text { Parques científico-tecnológicos } \\
\text { Contratación de graduados } \\
\text { Formación }\end{array}$ \\
\hline $\begin{array}{l}\text { Barge-Gil } \\
\text { y Modrego } \\
\text { (2011) }\end{array}$ & 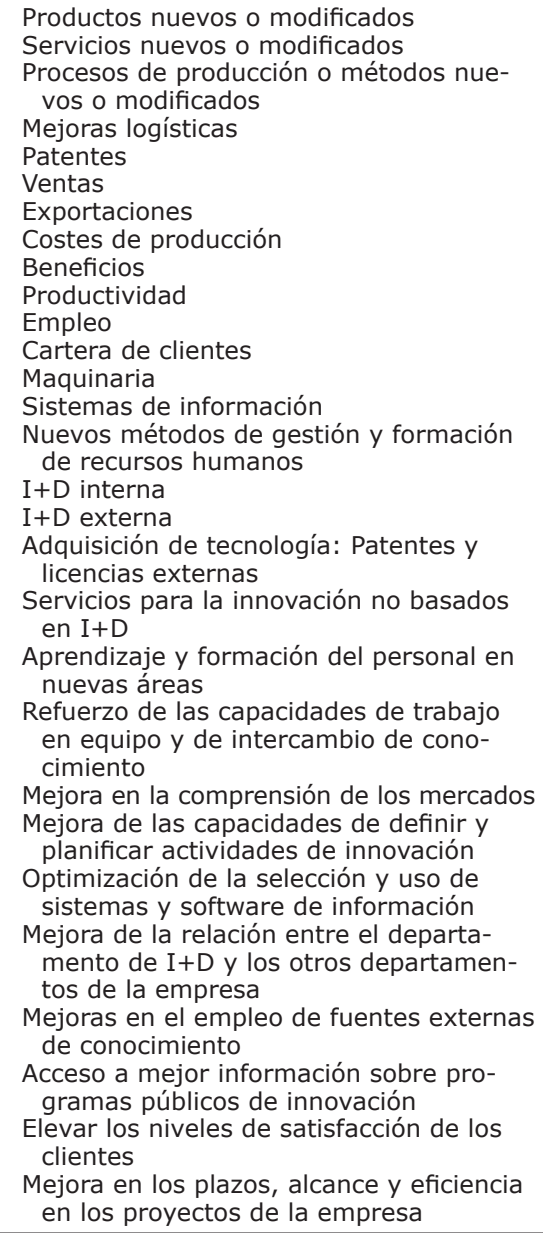 & $\begin{array}{l}\text { De Fuentes } \\
\text { y Dutrénit } \\
(2012)\end{array}$ & $\begin{array}{l}\text { Transferencia de tecnología desde las orga- } \\
\text { nizaciones } \\
\text { Test de productos y procesos } \\
\text { Incremento del control de calidad } \\
\text { Adquisición de I+D complementaria } \\
\text { Adquisición de I+D sustitutiva } \\
\text { Adquisición de conocimiento para la resolución } \\
\text { de problemas } \\
\text { Aumento de la capacidad de búsqueda y absor- } \\
\text { ción de información tecnológica } \\
\text { Adquisición de información sobre tendencias en } \\
\text { I+D } \\
\text { Acceso a recursos humanos cualificados } \\
\text { Uso de recursos disponibles en las organizacio- } \\
\text { nes públicas de investigación }\end{array}$ \\
\hline
\end{tabular}


Tabla III. Distribución de empresas de la muestra según la comunidad autónoma en la que se ubica

\begin{tabular}{|l|c|}
\hline Comunidad Autónoma & Porcentaje de la muestra \\
\hline Madrid & 35 \\
\hline Cataluña & 22 \\
\hline Andalucía & 13 \\
\hline Comunidad Valenciana & 5 \\
\hline País Vasco & 5 \\
\hline Castilla León & 4 \\
\hline Galicia & 3 \\
\hline Resto de comunidades & 13 \\
\hline
\end{tabular}

Para la identificación de las personas que pueden responder a la encuesta se ha partido de los nombres de los signatarios o de las personas de contacto indicadas en el contrato o convenio suscrito con el CSIC. En primer lugar, se realiza un proceso de identificación de las personas a entrevistar mediante llamada telefónica. Una vez identificadas estas personas, se les envía una copia del cuestionario, junto a una descripción breve de los objetivos del estudio. Posteriormente, se establece una cita con esta persona y un encuestador profesional se desplaza a la sede de la empresa para realizar la entrevista y, en su caso, responder dudas y validar las respuestas. El trabajo de campo se ha efectuado entre el 1 de octubre de 2010 y el 31 de enero de 2011.

Entre los perfiles profesionales de las personas a las que se ha entrevistado predominan los cargos directivos y los responsables de los departamentos de I+D.

Las empresas que mantienen relaciones contractuales con el CSIC forman un grupo muy diverso que tienen pocos puntos en común con la media de empresas existentes en España. En su mayoría son grandes y medianas empresas $(52,7 \%)$ mientras que este tipo de empresas representan un 2,38\% del total del tejido empresarial español (Directorio Central de Empresas (DIRCE), 2014). Buena parte de las empresas analizadas desarrollan sus actividades en sectores industriales de alta y media-alta tecnología $(17,9 \%)$ frente al $1,44 \%$ en el conjunto de empresas españolas. En lo que respecta al sector servicios, el $14,4 \%$ de las empresas consultadas pertenecen a la categoría de servicios de alta tecnología, cuando en el conjunto del tejido empresarial español este porcentaje se reduce al 2,08\% (DIRCE, 2014). La mayoría de las empresas de la muestra facturan en el mercado internacional $(55,9 \%)$, siendo en el grupo total de empresas españolas un $20 \%$ las que operan en mercados inter- nacionales (Instituto de Comercio Exterior (ICEX), 2013). Por tanto, las organizaciones empresariales objeto de estudio son mucho más grandes, de mayores niveles tecnológicos y más internacionalizadas de lo habitual en España. En la tabla IV se exponen los rasgos generales de las empresas que conforman la muestra de estudio.

El diseño del cuestionario ha estado guiado teóricamente de acuerdo con las principales líneas de investigación referidas a motivaciones, mecanismos y beneficios de la interacción. Se han incluido preguntas específicas referidas a cada una de las dimensiones, empleando una serie de indicadores para los que se ha solicitado a las empresas su posicionamiento a través de escalas tipo likert.

Para cada una de las dimensiones se ha incluido un amplio número de ítems que reflejan la diversidad apuntada por la investigación especializada. Ello permite obtener una descripción precisa de la realidad y, a la vez, contrastar las conclusiones sugeridas por la literatura para el caso de las empresas españolas que se relacionan con el CSIC.

\section{RESULTADOS Y DISCUSIÓN}

\subsection{Motivaciones de las empresas para interac- cionar con el CSIC}

Sobre la base de las aportaciones de los estudios económicos sobre cooperación tecnológica analizados, hemos estructurado las posibles razones que conducen a las empresas a relacionarse con un organismo público como el CSIC en dos grupos principales: las motivaciones tácticas y las motivaciones estratégicas.

En el presente trabajo de investigación se plantean un total de diecinueve cuestiones sobre las motivaciones tácticas y estratégicas que pueden influir en la decisión de las empresas de relacionarse con los institutos del CSIC. La medición de estas 
Tabla IV. Características de las empresas de la muestra

\begin{tabular}{|c|c|c|c|}
\hline & & Frecuencia & Porcentaje (\%) \\
\hline \multirow{9}{*}{ Sector de actividad } & Sectores de tecnología alta & 64 & 8,1 \\
\hline & Sectores de tecnología media-alta & 77 & 9,8 \\
\hline & Sectores de tecnología baja & 154 & 19,6 \\
\hline & Sector primario & 2 & 0,3 \\
\hline & Servicios avanzados a empresas & 113 & 14,4 \\
\hline & Otros Servicios & 103 & 13,1 \\
\hline & Otros & 64 & 8,1 \\
\hline & $\mathrm{NS} / \mathrm{NC}$ & 77 & 9,8 \\
\hline & Total & 787 & 100,0 \\
\hline \multirow{6}{*}{ Tamaño empresarial } & Grande & 109 & 18,8 \\
\hline & Mediana & 182 & 33,9 \\
\hline & Pequeña & 267 & 23,1 \\
\hline & Microempresa & 148 & 13,9 \\
\hline & NS/NC & 81 & 10,3 \\
\hline & Total & 787 & 100,0 \\
\hline \multirow{4}{*}{ Mercado internacional } & Sí & 440 & 55,9 \\
\hline & No & 228 & 29,0 \\
\hline & NS/NC & 119 & 15,1 \\
\hline & Total & 787 & 100,0 \\
\hline
\end{tabular}

motivaciones se realiza de dos formas alternativas. Por una parte se utiliza una escala de cuatro puntos cuyos extremos son "nada importante" y "muy importante", para medir el grado de importancia de cada una de las diecinueve motivaciones (véase Tabla V). Por otra parte, para forzar a las empresas a seleccionar las motivaciones más importantes se les pide que señalen las tres razones más decisivas para la interacción con el CSIC (véase Figura 1).

Las dos motivaciones que las empresas, en más ocasiones, reconocen como bastante o muy importantes para vincularse con el CSIC son, por un lado, mejorar la posición competitiva $y$, por otro lado, introducir nuevos productos o servicios en el mercado. En concreto, en torno al $70 \%$ de las empresas consideran estas dos razones como bastante o muy importantes.

En un segundo nivel de importancia, reconocidas como bastante o muy importantes por alrededor del $60 \%$ de las empresas, éstas se decantan por adelantarse a los cambios tecnológicos, acceder a redes de conocimiento científico-técnico no disponibles en el sector empresarial, y abordar proyectos de mayor complejidad, lo que pone de relieve la necesidad que existe en estas empresas de incorporar a su actividad un conocimiento específi- co que pueden obtener a través del CSIC. A este mismo nivel se sitúa una motivación de marketing: mejorar la imagen y prestigio de la empresa por relacionarse con el CSIC.

En un tercer nivel de importancia, señaladas como bastante o muy importantes por alrededor del $50 \%$ de las empresas, se encuentran aprender nuevas habilidades y capacidades y conocer el estado del arte en una tecnología o campo científico, al igual que la posibilidad de acceder a instalaciones o equipamiento de la organización de investigación, única motivación no estratégica que aparece entre las motivaciones más sentidas por las empresas para relacionarse con el CSIC.

Por el contrario, la colaboración de las empresas con el CSIC no está motivada en primer término por aspectos como la subcontratación de actividades de $I+D$ o la reducción de tiempos para la innovación, así lo confirman el $56 \%$ y $60 \%$ de las empresas consultadas respectivamente. Del mismo modo la obtención de financiación pública adicional, la reducción de riesgos y costes asociados a la I+D, o bien la búsqueda de una solución a un problema productivo urgente para la empresa tampoco se encuentran entre los principales objetivos perseguidos por las empresas. En concreto, se 
Tabla V. Motivaciones para relacionarse con el CSIC y grado de importancia atribuida

\begin{tabular}{|c|c|c|c|c|c|c|c|c|}
\hline & & \multicolumn{2}{|c|}{ Nada/Poco } & \multicolumn{2}{|c|}{$\begin{array}{l}\text { Bastante/ } \\
\text { Mucho }\end{array}$} & \multicolumn{2}{|c|}{ NS/NC } & \multirow{2}{*}{$\frac{\text { Total }}{\mathbf{N}}$} \\
\hline & & $\mathbf{N}$ & $\%$ & $\mathbf{N}$ & $\%$ & $\mathbf{N}$ & $\%$ & \\
\hline \multirow{12}{*}{$\begin{array}{l}\text { Motivaciones } \\
\text { tácticas }\end{array}$} & Introducir nuevos productos o servicios en el mercado & 228 & 29,0 & 518 & 65,8 & 41 & 5,2 & 787 \\
\hline & Acceder a nuevos mercados & 380 & 48,3 & 360 & 45,7 & 47 & 6,0 & 787 \\
\hline & Crear o hacer viable una nueva empresa & 653 & 83,0 & 73 & 9,3 & 61 & 7,8 & 787 \\
\hline & Solucionar un problema productivo urgente para la empresa & 540 & 68,6 & 195 & 24,8 & 52 & 6,6 & 787 \\
\hline & Subcontratar las actividades de I+D & 439 & 55,8 & 295 & 37,5 & 53 & 6,7 & 787 \\
\hline & Reducir riesgos y costes asociados a la $\mathrm{I}+\mathrm{D}$ & 527 & 67,0 & 199 & 25,3 & 61 & 7,8 & 787 \\
\hline & Reducir tiempos para la innovación & 478 & 60,7 & 251 & 31,9 & 58 & 7,4 & 787 \\
\hline & Obtener ventajas fiscales & 607 & 77,1 & 113 & 14,4 & 67 & 8,5 & 787 \\
\hline & Obtener financiación pública & 508 & 64,5 & 222 & 28,2 & 57 & 7,2 & 787 \\
\hline & Acceder a instalaciones o equipamiento del CSIC & 369 & 46,9 & 370 & 47,0 & 48 & 6,1 & 787 \\
\hline & Acceder a contactos para contratar personal & 622 & 79,0 & 107 & 13,6 & 58 & 7,4 & 787 \\
\hline & $\begin{array}{l}\text { Acceder a una patente o propiedad intelectual de la que el } \\
\text { CSIC es titular }\end{array}$ & 653 & 83,0 & 73 & 9,3 & 61 & 7,8 & 787 \\
\hline \multirow{7}{*}{$\begin{array}{l}\text { Motivaciones } \\
\text { estratégicas }\end{array}$} & Mejorar la posición competitiva & 212 & 26,9 & 535 & 68,0 & 40 & 5,1 & 787 \\
\hline & Mejorar la imagen y prestigio de la empresa & 299 & 38,0 & 449 & 57,1 & 39 & 5,0 & 787 \\
\hline & Adelantarse a los cambios tecnológicos & 267 & 33,9 & 474 & 60,2 & 46 & 5,8 & 787 \\
\hline & Abordar proyectos de mayor complejidad & 263 & 33,4 & 484 & 61,5 & 40 & 5,1 & 787 \\
\hline & $\begin{array}{l}\text { Acceder a redes de conocimiento científico-técnicos no dis- } \\
\text { ponibles en el sector empresarial }\end{array}$ & 271 & 34,4 & 473 & 60,1 & 43 & 5,5 & 787 \\
\hline & Conocer el estado del arte en una tecnología o campo científico & 364 & 46,3 & 372 & 47,3 & 51 & 6,5 & 787 \\
\hline & Aprender nuevas habilidades y capacidades & 344 & 43,7 & 393 & 49,9 & 50 & 6,4 & 787 \\
\hline
\end{tabular}

trata de objetivos considerados como nada o poco importantes para dos tercios de las empresas. Menos importancia atribuyen aún a la obtención de posibles ventajas fiscales, considerada por el $77 \%$ de las empresas como nada o poco importante.

Todos estos motivos, a los cuales las empresas asignan poca importancia para relacionarse con el CSIC, son de naturaleza táctica, mientras que los más valorados, con una única excepción, son los de índole estratégica. Ello indica que las empresas que se relacionan con el CSIC conocen bien la naturaleza del organismo, lo que pone así mismo de manifiesto el que las empresas no trabajen con el CSIC con la expectativa de futuras contrataciones de personal, tal y como lo demuestra el $79 \%$ de los casos analizados, aspecto éste que marca una diferencia reseñable con respecto a las universidades, que representan para las empresas una vía importante para la contratación de personal cualificado.

Hay dos motivaciones estratégicas que no constituyen motivaciones de peso para interaccionar con el CSIC, la creación de nuevas empresas o licenciar una patente de la que es titular el CSIC. Las empresas indican que ambos son motivos nada o poco importantes en el $83 \%$ de los casos.

En la selección de las principales motivaciones para relacionarse con el CSIC de la figura 1 se incluyen los resultados de una pregunta con opción de respuesta múltiple, en la que se requiere a la empresa que indique los tres motivos más importantes por los que ha decidido trabajar con el CSIC.

En primer lugar, aparecen dos motivaciones que destacan sobre las demás: introducir nuevos productos o servicios en el mercado y mejorar la posición competitiva. En segundo lugar, a una distancia significativa de estas dos y de las otras motivaciones se sitúan abordar proyectos de mayor complejidad y adelantarse a los cambios tecnológicos. En definitiva, lo que las empresas consultadas esperan de sus relaciones con el CSIC es que la institución les acompañe en sus procesos de innovación y en aquellos proyectos que deben afrontar con conocimiento de fuentes externas, ya sea 
Figura 1. Principales motivaciones para la interacción con el CSIC

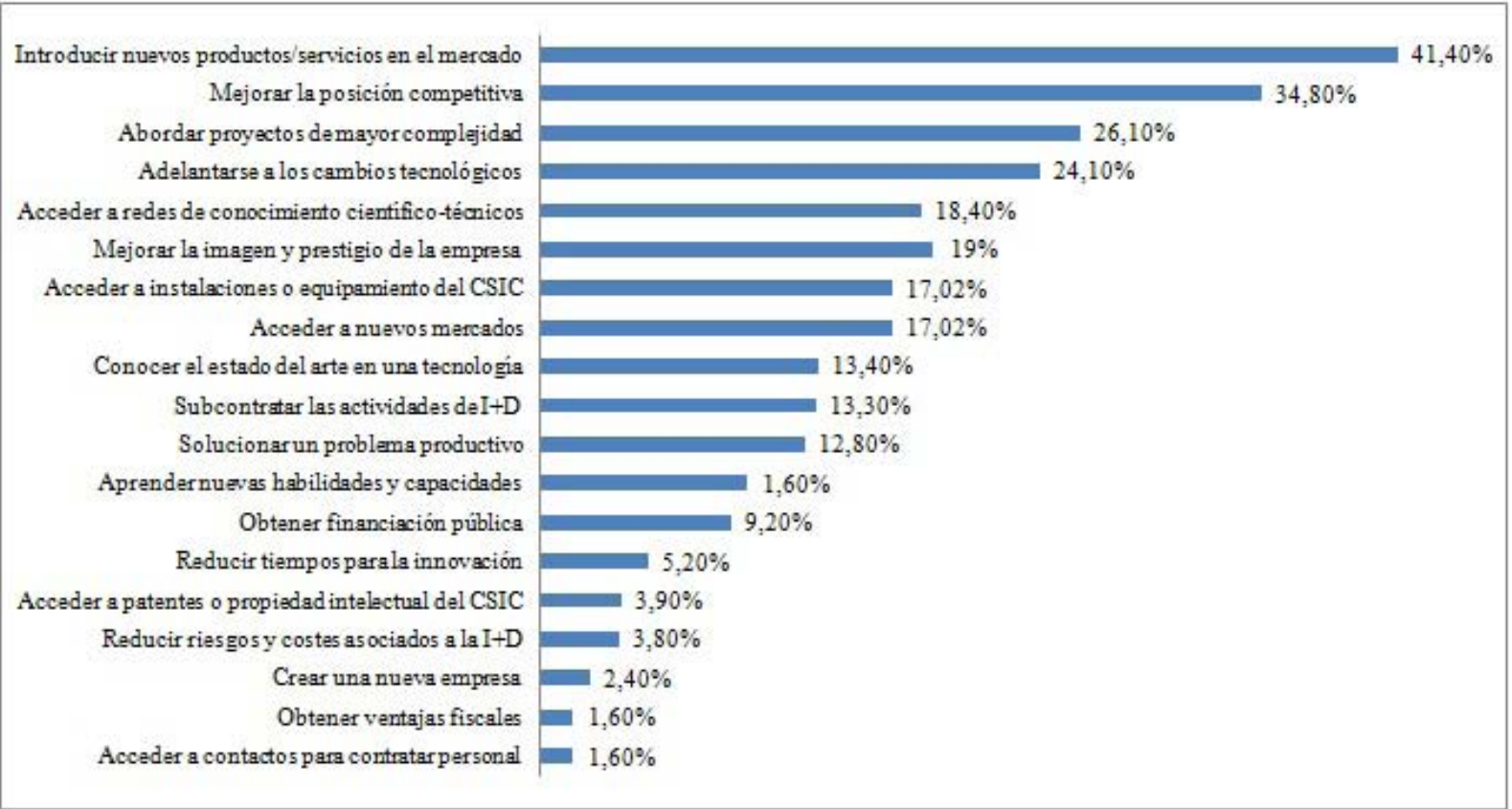

para acelerar la introducción de nuevos productos o servicios, para poder anticipar los cambios que afecten a una determinada tecnología, identificar aplicaciones adicionales de dicha tecnología o abordar retos de mayor complejidad que, en última instancia, puedan fortalecer la posición competitiva de la empresa en el mercado. Estas motivaciones son todas ellas estratégicas, es decir, se sitúan a medio y largo plazo en la estrategia de la empresa, y corroboran los resultados obtenidos precedentemente sobre las motivaciones de las empresas para relacionarse con el CSIC.

Asimismo, un quinto de las empresas también trabajan con el CSIC con el objeto de mejorar su imagen y prestigio, lo que denota el prestigio de este organismo de investigación como proveedor de recursos científicos entre los agentes del SI español.

De forma consistente con la literatura, estos resultados otorgan un papel clave a esta institución de investigación para que las empresas alcancen objetivos estratégicos -o de exploración-, es decir, las empresas estudiadas buscan en el CSIC recursos y capacidades complementarias que les permita mantener y reforzar su posición competitiva (Hagedoorn y otros, 2000). En menor medida, destacan la escasa importancia que la interacción con un organismo como el CSIC tiene para la consecución de objetivos tácticos -o de explotación- aunque algunos de ellos sean considerados útiles en algunos casos como la subcontratación de actividades de I+D o la solución de problemas urgentes para las empresas. Estos resultados van en el sentido de los obtenidos por un segmento de la literatura que atribuye un papel preponderante en la innovación empresarial a otro tipo de agentes debido a su profundo conocimiento de las necesidades del mercado y su mayor proximidad al mismo: organizaciones empresariales de la cadena de valor, como clientes y proveedores (Tether, 2002; Bayona y otros, 2003) o centros tecnológicos (Olazaran y otros, 2009; 2011).

El papel jugado por el CSIC a la luz de los resultados expuestos en este trabajo puede ser interpretado a partir del estudio de la fase del proceso de innovación en la que interviene cada uno de los agentes (Fritsch y Schwirten, 1999; Schartinger y otros, 2002; Wright y otros, 2008; Cassiman y otros, 2010). En este sentido, la contribución del CSIC en la actividad innovadora se focaliza en las fases iniciales del proceso de innovación, cuando es necesario generar nuevo conocimiento que, posteriormente, puede ser utilizado para obtener una innovación.

Sin embargo, otras motivaciones tradicionalmente señaladas en la literatura, como el ahorro en los costes asociados a las actividades de investigación 
(Bonaccorsi y Piccaluga, 1994) o la obtención de recursos económicos para financiar proyectos (Bayona y otros, 2002) son poco valoradas por las empresas que se han relacionado con el CSIC. Tampoco los resultados obtenidos avalan la hipótesis sostenida en investigaciones previas (De Fuentes y Dutrénit, 2012; Merchán-Hernández, 2012) de que la interacción con agentes científicos permite identificar personal cualificado y con especialización técnica para futuras contrataciones en las empresas. Las empresas españolas no parecen perseguir ese fin en su relación con el CSIC.

\subsection{Mecanismos de interacción utilizados entre las empresas españolas y el CSIC}

La diversidad de motivaciones por las que las empresas se relacionan con el CSIC ha producido que este organismo adopte diferentes mecanismos para adaptarse a la variedad de posibles relaciones. Los resultados indican que los mecanismos más utilizados por las empresas son los de apoyo tecnológico o consultoría, como así se demuestra en el $65 \%$ de las observaciones, seguido de la investigación contratada en el $52 \%$ de los casos. A muy poca distancia aparecen los contactos informales. Esta distribución en la utilización de los mecanismos es prácticamente idéntica a la encontrada en otras encuestas a empresas españolas que se relacionan con los agentes científicos del SI, ver a este respecto García-Aracil y Fernándezde-Lucio (2008). En un escalón más bajo se sitúa la investigación en el marco de programas públicos de carácter estatal y el uso de las instalaciones o equipos del CSIC, con el $42 \%$ de las respuestas.
Asimismo, la participación conjunta en actividades de difusión es una forma de vinculación presente en el $31 \%$ de las empresas estudiadas. Este resultado es inesperado por su magnitud y novedoso pues no suele preguntarse en este tipo de encuestas (tabla VI).

Estos resultados son coincidentes con los alcanzados por otros investigadores que identifican un escenario de interacción que da cabida a formas de relación variadas (Agrawal, 2001; Cohen y otros., 2002; D'Este y Patel, 2007; Perkmann y Walsh, 2007; Bekkers y Boda-Freitas, 2008; Arza, 2010; Merchán-Hernández, 2012; García-Carpintero y otros, 2014).

Las actividades relacionadas con la formación no presentan frecuencias muy elevadas. Únicamente en el $8 \%$ de los casos se afirma haber formado a posgraduados en las empresas. Algo más elevado es el porcentaje de casos en los que el CSIC ha ofrecido formación especializada a la empresa, en el $17 \%$ de los casos. En cuanto a estancias de personal de las empresas en institutos del CSIC, esta forma de transferencia de conocimiento se ha realizado en el $13 \%$ de los casos consultados. Estos resultados denotan que, frente a la labor formadora que realizan las universidades como parte de su misión, el papel del CSIC puede ser catalogado de modesto.

Los datos evidencian, una vez más, que las licencias de patentes $u$ otro título de propiedad intelectual y la creación de nuevas empresas en colaboración tienen escasa presencia en las relaciones entre las empresas y el CSIC.

Tabla VI. Mecanismos utilizados por las empresas en su relación con el CSIC

\begin{tabular}{lccc}
\hline & N & $\begin{array}{c}\text { \% } \\
\text { Muestra }\end{array}$ & $\begin{array}{c}\text { \% } / \text { NC } \\
\text { NS }\end{array}$ \\
\hline Licencia de patentes (u otras modalidades de protección de propiedad intelectual) & 97 & 12,3 & 4,2 \\
\hline Creación de una nueva empresa en colaboración & 18 & 2,30 & 3,7 \\
\hline Apoyo tecnológico o consultoría & 509 & 64,7 & 3,6 \\
\hline Uso de instalaciones o equipos del CSIC & 336 & 42,7 & 3,7 \\
\hline Investigación contratada & 410 & 52,1 & 4,2 \\
\hline Investigación en el marco de un programa público & 331 & 42,1 & 4,6 \\
\hline Investigación en el marco de programas internacionales & 108 & 13,7 & 5,0 \\
\hline Estancias del personal de su empresa en el CSIC & 105 & 13,3 & 3,9 \\
\hline Formación de posgraduados en su empresa (incluyendo tesis doctorales) & 62 & 7,90 & 3,9 \\
\hline Personal de su empresa ha recibido formación especializada por parte del CSIC & 135 & 17,2 & 3,7 \\
\hline Participación conjunta en actividades de difusión & 243 & 30,9 & 3,9 \\
\hline Contactos o consultas no formalizadas en un contrato o acuerdo & 378 & 48,0 & 4,1 \\
\hline
\end{tabular}


En cuanto a la importancia que las empresas atribuyen a las actividades que realizan con los institutos del CSIC, hay que señalar que todas son importantes sin diferencias reseñables. Esto significa que, una vez que las empresas establecen relaciones con la institución investigadora, la relevancia de éstas es considerable. Prueba de ello es que todos los mecanismos de interacción son calificados como bastante o muy importantes por las empresas, con porcentajes superiores al $75 \%$. A priori no es posible discriminar entre los mecanismos de interacción atendiendo a su importancia en la actividad empresarial.

\subsection{Beneficios de las relaciones entre las em- presas españolas y el CSIC}

Uno de los ámbitos donde es posible observar el efecto de las relaciones entre las empresas y los institutos del CSIC es el técnico. En este sentido, los resultados obtenidos ratifican el papel del CSIC como agente que favorece la innovación empresarial. En el $41,7 \%$ de los casos se verifica que el lanzamiento al mercado de un nuevo producto o servicio se ha visto favorecido por la interacción con el CSIC y el 55\% de las empresas aseguran haber introducido alguna mejora en su producto o servicio. Por otro lado, un porcentaje considerable de empresas, el 48,4\%, asegura haber resuelto un problema técnico en la empresa como resultado de la relación (tabla VII).

La generación de una patente o título de propiedad intelectual es un resultado que aparece en el $18 \%$ de las empresas entrevistadas, porcentaje significativo, que indica que la creación de nuevo conocimiento en estas interacciones es importante y abre una vía de generación de patentes en colaboración que debería profundizar el CSIC.

Las empresas afirman haber mejorado su cifra de beneficio como consecuencia de su relación con el CSIC en un porcentaje elevado de los casos, el $37 \%$, siendo este resultado, entre los de tipo económico, el más destacado entre los considerados. Esto quiere decir que así como los intercambios de conocimientos son diversos, sus beneficios se distribuyen en diferentes actividades de la empresa y el indicador que mejor recoge el conjunto es el beneficio empresarial. Una de las bondades tradicionalmente atribuidas a la interacción entre agentes es que permite reducir los riesgos y costes asociados a las actividades de I+D, sin embargo, el estudio solo confirma una moderada presencia del resultado mencionado, concretamente en el $29 \%$ de los casos estudiados (tabla VIII).

Tabla VII. El efecto de la interacción en el ámbito técnico

\begin{tabular}{lccc}
\hline Como consecuencia de la interacción ha sido posible: & Frec. & \% & \% válido \\
\hline Lanzar un nuevo producto o servicio & 328 & 41,7 & 43,4 \\
\hline Mejorar un producto o servicio & 437 & 55,5 & 57,7 \\
\hline Obtener un nuevo proceso productivo de bienes o servicios & 168 & 21,3 & 22,3 \\
\hline Mejorar un proceso productivo & 227 & 28,8 & 30,0 \\
\hline Mejorar prácticas empresariales o procedimientos de trabajo & 188 & 23,9 & 25,0 \\
\hline Conseguir asistencia en la resolución de problemas & 381 & 48,4 & 50,7 \\
\hline Generar una patente o propiedad intelectual & 143 & 18,2 & 19,2 \\
\hline $\begin{array}{l}\text { Nota: La columna \% considera como base las 787 empresas consultadas. La columna \% válido utiliza como base el total de las } \\
\text { empresas que responden al ítem en cuestión, es decir, se excluyen las empresas que responden NS/NC. En adelante se aplica } \\
\text { el mismo significado. }\end{array}$ &
\end{tabular}

Tabla VIII. El efecto de la interacción de índole económico

\begin{tabular}{lccc}
\hline Como consecuencia de la cooperación ha sido posible: & Frec. & $\%$ & \% válido \\
\hline Mejorar el beneficio económico & 292 & 37,1 & 39,6 \\
\hline Mejorar la cifra de exportaciones & 113 & 14,4 & 15,3 \\
\hline Acceder a un nuevo mercado & 232 & 29,5 & 30,8 \\
\hline Reducir riesgos y costes asociados a la I+D. & 229 & 29,1 & 30,7 \\
\hline Crear una nueva empresa & 43 & 5,5 & 5,7 \\
\hline
\end{tabular}


La interacción con el CSIC ha tenido un claro efecto en la decisión de potenciar la inversión de la empresa en I+D. El $43 \%$ de las empresas aseveran haber incrementado su inversión en I+D como resultado de su relación con el CSIC, si bien, esta inversión tiene un menor reflejo en la estructura organizativa de las empresas, pues tan solo el $17,3 \%$ de las empresas han creado o aumentado el departamento de I+D. Aún son menos las que han incrementado su plantilla, únicamente el $13,1 \%$ de las empresas consultadas (tabla IX).

En cuanto al grupo de resultados clasificados como de índole intangible, se advierte que la adquisición de conocimiento científico-técnico es el logro más frecuente entre las empresas estudiadas. El $71 \%$ de las empresas declaran haber adquirido nuevos conocimientos científico-técnicos como resultado de su vinculación con el CSIC, lo que confirma el estratégico papel de la institución como proveedor del conocimiento técnico que requieren las empresas para el desarrollo de su actividad. A esto hay que añadir que las empresas no solo reciben conocimiento técnico del CSIC, sino también conocimiento para identificar nuevas oportunidades de negocio, que si bien su transferencia ocurre en menos ocasiones, es sin duda significativo el porcentaje del $38,2 \%$ de empresas que han conseguido ampliar su modelo de negocio en el marco de sus relaciones con el CSIC (tabla $X$ ).

Merece ser destacado, asímismo, que el 62\% de las empresas consideran que su imagen y prestigio se han visto favorecidos en el mercado por el hecho de haber trabajado con el CSIC. Se trata, por tanto, de uno de los beneficios más frecuentes entre las empresas $y$, a su vez, uno de los mejor valorados.

Un aspecto que resulta crucial para la competitividad empresarial es la cualificación de los recursos humanos, en la medida en que ésta se relaciona directamente con la capacidad de identificar, absorber y aplicar el conocimiento de agentes externos. En este sentido, la interacción con el CSIC ha permitido desarrollar la cualificación de los recursos humanos y aumentar la cultura innovadora a nivel interno, como lo afirman respectivamente el $42 \%$ y el $50 \%$ de las empresas. Un resultado que pone de manifiesto que la interacción con el personal investigador del CSIC supone una fuente de aprendizaje importante para el personal de las empresas, y que la colaboración conforma un formidable escenario para el intercambio, tanto de conocimiento explícito, como tácito y, además, el desarrollo competencial del capital humano.

Otro de los aspectos que a menudo se ve reforzado como resultado de la interacción con agentes científicos, es el capital social de la empresa. Los datos de la tabla $X$, apoyan este planteamiento. EI CSIC, fundamentalmente, contribuye con contactos de universidades y otros centros públicos de investigación en el $43 \%$ de los casos estudiados. Aunque de manera menos destacada, la interacción también facilita el contacto con otro tipo de agentes, como consultoras, centros tecnológicos o laboratorios privados de I+D, y con otras empresas privadas. El $25,2 \%$ y $27,1 \%$ de las empresas respectivamente afirman haber incrementado sus contactos con los agentes mencionados.

La amplia gama de beneficios detectados en la presente investigación va en la línea de recientes trabajos que han evidenciado la naturaleza multidimensional y compleja del impacto de la interacción entre los agentes empresariales y científicos. Tal y como sugieren Barge-Gil y Modrego (2011), los efectos de la relación entre el sector empresarial y el científico pueden observarse en el plano técnico, económico, en la inversión empresarial y en una vertiente más intangible. El presente estudio confirma beneficios para las empresas analizadas en todos los ámbitos anticipados por el citado referente empírico.

Tabla IX. El efecto de la interacción relacionado con el incremento de inversión en la empresa

\begin{tabular}{lccc}
\hline Como consecuencia de la cooperación ha sido posible: & Frec. & $\%$ & $\%$ válido \\
\hline Aumentar la inversión en I+D en la empresa & 337 & 42,8 & 45,1 \\
\hline Contratar nuevo personal & 103 & 13,1 & 13,8 \\
\hline Crear o aumentar el departamento de I+D & 136 & 17,3 & 18,2 \\
\hline
\end{tabular}


Tabla X. El efecto de la interacción de tipo intangible

\begin{tabular}{|c|c|c|c|}
\hline Como consecuencia de la cooperación ha sido posible: & Frec. & $\%$ & \% válido \\
\hline Mejorar la imagen y prestigio & 493 & 62,6 & 65,6 \\
\hline Aumentar la cultura innovadora en la empresa & 391 & 49,7 & 52,4 \\
\hline Obtener conocimiento para identificar nuevas oportunidades de negocio & 301 & 38,2 & 40,1 \\
\hline Adquirir conocimientos científico-técnicos & 557 & 70,8 & 73,7 \\
\hline Mejorar la cualificación del personal & 331 & 42,1 & 43,9 \\
\hline Mejorar las capacidades de gestión de proyectos o de actividades de innovación & 189 & 24,0 & 25,3 \\
\hline Establecer nuevos contactos con universidades y otros centros públicos de investigación & 338 & 42,9 & 45,2 \\
\hline $\begin{array}{l}\text { Establecer nuevos contactos con consultoras, centros tecnológicos o laboratorios privados } \\
\text { de I+D }\end{array}$ & 198 & 25,2 & 26,5 \\
\hline $\begin{array}{l}\text { Establecer nuevos contactos con otras empresas (e.j. competidores, potenciales clientes, } \\
\text { potenciales proveedores) }\end{array}$ & 213 & 27,1 & 28,6 \\
\hline
\end{tabular}

\section{CONCLUSIONES}

Este trabajo contribuye empíricamente en tres aspectos fundamentales al estudio de la relaciones entre las empresas y el sector científico: primero, qué motivaciones conducen a las empresas a relacionarse con el CSIC; segundo, qué mecanismos sustentan la relación entre dichos agentes, y tercero y último, qué beneficios han obtenido las empresas como consecuencia de dicha interacción, permitiendo efectuar una evaluación del efecto de la interacción a partir del amplio y diverso conjunto de indicadores. Este estudio presenta, por tanto, implicaciones para las empresas y para los decisores públicos.

Las motivaciones que llevan a las empresas a relacionarse con el CSIC son variadas, ya que han sido analizadas empresas de todos los sectores y tamaños; sin embargo, las empresas prefieren y valoran mejor, en general, las motivaciones estratégicas que las tácticas. Una motivación que indica el peso del CSIC en el sistema científico es el hecho de que una mayoría de empresas considera que relacionarse con el CSIC mejora su imagen y prestigio.

Hay, sin embargo, motivaciones poco valoradas como obtener ventajas fiscales o lograr financiación pública, que los policy makers y gestores de las relaciones sobrevaloran, del mismo modo que sucede con crear o hacer viable una empresa o acceder a una patente o propiedad intelectual del CSIC. Sin embargo, las empresas aprecian bastante acceder a las instalaciones o equipamientos del CSIC, argumento que debiera ser valorizado por los gestores del organismo público.

En correspondencia con esta diversidad de motivaciones, los mecanismos que articulan la rela- ción entre los agentes son también diversos. Los resultados obtenidos destacan que los mecanismos de interacción más utilizados por las empresas son los de apoyo tecnológico o consultoría y los de investigación contratada, mecanismos de bajo nivel de sofisticación. El uso de instalaciones o equipos del CSIC o la investigación en el marco de un programa público son también bastante utilizados. A la vista de estos resultados, que son coincidentes con los encontrados por otros investigadores en otras encuestas a empresas españolas, sorprende que los policy makers o gestores de las relaciones sientan tanta inclinación por la puesta en práctica de mecanismos muy sofisticados como la creación de centros mixtos o de empresas mixtas, no se sabe muy bien si es por una mala adaptación de modelos foráneos de territorios muy diferentes al español o por el desconocimiento de las necesidades de las empresas españolas. En este sentido, los resultados obtenidos, entre los que destacan la alta valoración que las empresas otorgan a los contactos informales, nos lleva a la conclusión que, en el caso español, los mecanismos más apropiados son aquellos que facilitan y favorecen en mayor medida el que se puedan producir estas relaciones.

Los beneficios que las empresas obtienen de su interacción se extienden más allá de los efectos sobre la innovación y, en consecuencia, sobre los beneficios económicos asociados a la misma, y cubren una serie de beneficios intangibles, algunos sutiles como la mejora de su imagen y prestigio en el mercado por el hecho de haber trabajado con un organismo como el CSIC. De igual forma, la interacción entre estos agentes actúa como elemento catalizador del aumento de la inversión de las empresas en actividades de I+D, como también, el flujo de conocimiento 
intercambiado ha dejado su impronta en las empresas mejorando la cualificación de su personal. Esta amplia gama de beneficios justifica la dificultad histórica de hacer una evaluación precisa del impacto del CSIC, y de los OPI en general, en sus relaciones con las empresas.

Si bien la presente investigación supone un avance en el estudio de la interacción entre las empresas y los organismos públicos de investigación, existe, sin embargo, la necesidad de seguir avanzando en el análisis de los condicionantes que determinan la decisión de las empresas de interaccionar con estos agentes científicos, de comprobar si existe uno o varios patrones de desarrollo de la relación y de examinar con detalle qué aspectos influyen en el hecho de que las empresas alcancen en su relación con el CSIC unos resultados concretos.

\section{REFERENCIAS}

Agrawal, A. (2001). University-to-industry knowledge transfer: literature review and unanswered questions. International Journal of Management Reviews, vol. 3 (4), 285-302. http://dx.doi. org/10.1111/1468-2370.00069

Arza, V. (2010). Channels, benefits and risks of public-private interactions for knowledge transfer: conceptual framework inspired by Latin America. Science and Public Policy, vol. 37 (7), 473-484. http://dx.doi.org/10.3152/030234210X511990

Barge-Gil, A.; Modrego, A. (2011). The impact of research and technology organizations on firm competitiveness. Measurement and determinants. The Journal of Technology Transfer, vol. 36 (1), 6183. http://dx.doi.org/10.1007/s10961-009-9132-4

Bayona-Sáenz, C.; García-Marco, T.; Huerta-Arribas, E. (2001). Firms' motivations for cooperative R\&D: an empirical analysis of Spanish firms. Research Policy, vol. 30 (8), 1289-1307. http://dx.doi. org/10.1016/S0048-7333(00)00151-7

Bayona-Sáenz, C.; García-Marco, T.; Huerta-Arribas, E. (2002). Collaboration in R\&D with universities and research centres: an empirical study of Spanish firms. R\&D Management, vol. 32 (4), 321-341. http://dx.doi.org/10.1111/1467-9310.00264

Bayona, C.; García-Marco, T.; Huerta-Arribas, E. (2003). ¿Cooperar en I+ D? Con quién y para qué. Revista de Economía Aplicada, vol. 31(11), 103-134.

Becker, W.; Dietz, J. (2004). R\&D cooperation and innovation activities of firms-evidence for the German manufacturing industry. Research Policy, vol. 33 (2), 209-223. http://dx.doi.org/10.1016/j. respol.2003.07.003

\section{AGRADECIMIENTOS}

Los datos de este trabajo provienen del proyecto titulado "Una aproximación al impacto socioeconómico de las actividades del CSIC", realizado a instancias de la Presidencia del CSIC a través de la Vicepresidencia Adjunta de Transferencia de Conocimiento (VATC) y desarrollado conjuntamente entre el Instituto de Estudios Sociales Avanzados (IESA) y el Instituto de Gestión del Conocimiento y la Innovación (INGENIO). Los autores agradecen la aportación al proyecto de los investigadores/as Elena Castro, Pablo D'Este, Elena Espinosa, Antonio Gutiérrez, Liney Manjarrés, Carmen Merchán, Manuel Pérez Yruela, Leticia Rodríguez y Jaider Vega.

Los autores agradecen, asimismo, a los evaluadores y a la editora de la REDC por la atención dispensada y las sugerencias efectuadas en orden a mejorar el presente trabajo.

Bekkers, R.; Bodas-Freitas, I.M. (2008). Analysing knowledge transfer channels between universities and industry: To what degree do sectors also matter?. Research Policy, vol. 37 (10), 1837-1853. http://dx.doi.org/10.1016/j.respol.2008.07.007

Bishop, K.; D'Este, P.; Neely, A. (2011). Gaining from interactions with universities: Multiple methods for nurturing absorptive capacity. Research Policy, vol. 40 (1), 30-40. http://dx.doi.org/10.1016/j. respol.2010.09.009

Bonaccorsi, A.; Piccaluga, A. (1994). A theoretical framework for the evaluation of university-industry relationships. $R \& D$ Management, vol. 24 (3), 229247. http://dx.doi.org/10.1111/j.1467-9310.1994. tb00876.x

Bozeman, B.; Papadakis, M.; Coker, K. (1995). Industry Perspectives on Commercial Interactions with Federal Laboratories. National Science Foundation, Research on Science and Technology Program, Contract No. 9220125.

Cassiman, B.; Di Guardo, M.C.; Valentini, G. (2010). Organizing links with science: Cooperate or contract?: A project-level analysis. Research Policy, vol. 39 (7), 882892. http://dx.doi.org/10.1016/j.respol.2010.04.009

Cohen, W.; Nelson, R.; Walsh, J. (2002). Links and impacts: the influence of public research on industrial R\&D. Management Science, vol. 48 (1), 1-23. http://dx.doi.org/10.1287/mnsc.48.1.1.14273

D'Este, P.; Patel, P. (2007). University-industry linkages in the UK: What are the factors underlying the variety of interactions with industry?. Research Policy, vol. 36 (9), 1295-1313. http://dx.doi. org/10.1016/j.respol.2007.05.002 
De Fuentes, C.; Dutrénit, G. (2012). Best channels of academia-industry interaction for long-term benefit. Research Policy, vol. 41 (9), 1666-1682. http://dx.doi.org/10.1016/j.respol.2012.03.026

Di Gregorio, D.; Shane, S. (2003). Why do some universities generate more start-ups than others?. Research Policy, 32 (2), 209-227. http://dx.doi. org/10.1016/S0048-7333(02)00097-5

Directorio Central de Empresas (DIRCE) 2014. Datos de empresas. Madrid: Instituto Nacional de Estadística.

Edquist, C. (1997). Systems of Innovation: Technologies, Institutions and Organisations. Nueva York/Londres: Pinter Publishers.

Fritsch, M.; Schwirten, C. (1999). EnterpriseUniversity co-operation and the role of public research institutions in regional innovation systems. Industry and Innovation, vol. 6 (1), 69-83. http:// dx.doi.org/10.1080/13662719900000005

García-Aracil, A.; Fernández-de-Lucio, I. (2008). Industry-university interactions in a peripheral European region: an empirical study of Valencian firms. Regional Studies, vol. 42 (2), 215-227. http://dx.doi.org/10.1080/00343400601142704

García-Carpintero, E.; Albert-Martínez, A.; Granadino, B.; Plaza, L.M. (2014). Análisis de la colaboración entre las empresas biotecnológicas españolas con actividades de I+D y el sistema público de I+D. Revista Española de Documentación Científica, vol. 37 (2), e041. http://dx.doi.org/10.3989/ redc.2014.2.1106

Geisler, E. (2001). Explaining the generation and performance of intersector technology cooperation: a survey of the literature. Technology Analysis and Strategic Management, vol. 13 (2), 195-206. http://dx.doi.org/10.1080/09537320125169

Gutiérrez-Gracia, A.; Vega-Jurado, J.; Fernández-deLucio, I. (2010). Cooperación con agentes científicos y desempeño innovador. En: Sanz Menéndez, L.; Cruz Castro, L. (editores) Análisis sobre ciencia e innovación en España. FECYT.

Hagedoorn, J. (1993). Understanding the rationale of strategic technology partnering: Interorganizational modes of cooperation and sectoral differences. Strategic Management Journal, vol. 14 (5), 371371. http://dx.doi.org/10.1002/smj.4250140505

Hagedoorn, J.; Link, A.N.; Vonortas, N.S. (2000). Research partnerships. Research Policy, vol. 29 (4-5), 567-586. http://dx.doi.org/10.1016/S00487333(99)00090-6

ICEX España Exportación e Inversiones (2013). Madrid: Ministerio de Economía y Competitividad.

Jaffe, A. (1989). Real Effects of Academic Research. American Economic Review, vol. 79 (5), 957-970.
Lundvall, B.A. (1992). National systems of innovation: Towards a theory of innovation and interactive learning. London: Pinter.

Merchán-Hernández, C. (2012). Las relaciones de las empresas con las universidades: estrategias y dinámicas del proceso de cooperación a nivel regional. Arbor, vol. 188 (753), 193-209. http:// dx.doi.org/10.3989/arbor.2012.753n1012

Miotti, L.; Sachwald, F. (2003). Co-operative R\&D: why and with whom?: An integrated framework of analysis. Research Policy, vol. 32 (8), 1481-1499. http://dx.doi.org/10.1016/S0048-7333(02)00159-2

Mowery, D.C.; Ziedonis, A.A. (2002). Academic patent quality and quantity before and after the BayhDole Act in the United States. Research Policy, vol. 31 (3), 399-418. http://dx.doi.org/10.1016/ S0048-7333(01)00116-0

Narula, R. (2004). R\&D collaboration by SMEs: new opportunities and limitations in the face of globalization. Technovation, vol. 24 (2), 153-161. http://dx.doi.org/10.1016/s0166-4972(02)00045-7

Olazaran, M.; Albizu, E.; Otero, B. (2009). Technology transfer between technology centres and SMEs: Evidence from the Basque Country. European Planning Studies, vol. 17 (3), 345-363. http:// dx.doi.org/10.1080/09654310802618010

Olazaran, M.; Albizu, E.; Otero, B. (2011). Cooperación y creación de conocimiento tecnológico: Innovación en las pequeñas y medianas empresas industriales. En T. González de la Fe y M. Fernández, (Eds): Innovación tecnológica, conocimiento científico y cambio social, Centro de Investigaciones Sociológicas (CIS), Madrid, 131-162.

Perkmann, M.; Walsh, K. (2007). University-industry relationships and open innovation: Towards a research agenda. International Journal of Management Reviews, vol. 9 (4), 259-280. http:// dx.doi.org/10.1111/j.1468-2370.2007.00225.x

Ponomariov, B.; Boardman, C. (2012). Organizational Behavior and Human Resources Management for Public to Private Knowledge Transfer: An Analytic Review of the Literature. OCDE, Science, Technology and Industry Working Papers No. 2012/01. http:// dx.doi.org/10.1787/5k9d4gt7mdbp-en

Schartinger, D.; Rammer, C.; Fischer, M.M.; Frohlich, J. (2002). Knowledge interactions between universities and industry in Austria: sectoral patterns and determinants. Research Policy, vol. 31 (3), 303-328. http://dx.doi.org/10.1016/S00487333(01)00111-1

Tether, B.S. (2002). Who co-operates for innovation, and why: An empirical analysis. Research Policy, vol. 31 (6), 947-967. http://dx.doi.org/10.1016/ S0048-7333(01)00172-X 
Thursby, J.G.; Thursby, M.C. (2002). Who is selling the ivory tower? Sources of growth in university licensing. Management Science, vol. 48 (1), 90-104. http://dx.doi.org/10.1287/mnsc.48.1.90.14271

Vega-Jurado, J.; Gutiérrez-Gracia, A.; Fernándezde-Lucio, I. (2009). Does external knowledge sourcing matter for innovation? Evidence from the Spanish manufacturing industry. Industrial and Corporate Change, 18 (4), 637-670. http://dx.doi. org/10.1093/icc/dtp023
Veugelers, R. (1998). Collaboration in R\&D: an assessment of theoretical and empirical findings. The Economist, vol. 146 (3), 419-443. http:// dx.doi.org/10.1023/A:1003243727470

Wright, M.; Clarysse, B.; Lockett, A.; Knockaert, M. (2008). Mid-range universities' linkages with industry: Knowledge types and the role of intermediaries. Research Policy, vol. 37 (8), 1205-1223. http://dx.doi.org/10.1016/j. respol.2008.04.021 